\title{
Front Matter: Volume 9513
}

, "Front Matter: Volume 9513," Proc. SPIE 9513, High-Power, High-Energy, and High-Intensity Laser Technology II, 951301 (10 June 2015); doi:

10.1117/12.2197745

SPIE. Event: SPIE Optics + Optoelectronics, 2015, Prague, Czech Republic 


\title{
PROCEEDINGS OF SPIE
}

\section{High-Power, High-Energy, and High-Intensity Laser Technology II}

\author{
Joachim Hein \\ Editor
}

\author{
14-15 April 2015 \\ Prague, Czech Republic \\ Sponsored by \\ SPIE \\ Cooperating Organisations \\ HiPER Project (United Kingdom) \\ ELI Beamlines (Czech Republic) \\ Laserlab Europe
}

Published by

SPIE 
The papers included in this volume were part of the technical conference cited on the cover and title page. Papers were selected and subject to review by the editors and conference program committee. Some conference presentations may not be available for publication. The papers published in these proceedings reflect the work and thoughts of the authors and are published herein as submitted. The publisher is not responsible for the validity of the information or for any outcomes resulting from reliance thereon.

Please use the following format to cite material from this book:

Author(s), "Title of Paper," in High-Power, High-Energy, and High-Intensity Laser Technology II, edited by Joachim Hein, Proceedings of SPIE Vol. 9513 (SPIE, Bellingham, WA, 2015) Article CID Number.

ISSN: 0277-786X

ISBN: 9781628416343

Published by

SPIE

P.O. Box 10, Bellingham, Washington 98227-0010 USA

Telephone +1 3606763290 (Pacific Time) · Fax +1 3606471445

SPIE.org

Copyright (C) 2015, Society of Photo-Optical Instrumentation Engineers.

Copying of material in this book for internal or personal use, or for the internal or personal use of specific clients, beyond the fair use provisions granted by the U.S. Copyright Law is authorized by SPIE subject to payment of copying fees. The Transactional Reporting Service base fee for this volume is $\$ 18.00$ per article (or portion thereof), which should be paid directly to the Copyright Clearance Center (CCC), 222 Rosewood Drive, Danvers, MA 01923. Payment may also be made electronically through CCC Online at copyright.com. Other copying for republication, resale, advertising or promotion, or any form of systematic or multiple reproduction of any material in this book is prohibited except with permission in writing from the publisher. The CCC fee code is 0277-786X/15/\$18.00.

Printed in the United States of America.

Publication of record for individual papers is online in the SPIE Digital Library.

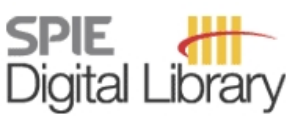

SPIEDigitalLibrary.org

Paper Numbering: Proceedings of SPIE follow an e-First publication model, with papers published first online and then in print. Papers are published as they are submitted and meet publication criteria. A unique citation identifier (CID) number is assigned to each article at the time of the first publication. Utilization of CIDs allows articles to be fully citable as soon as they are published online, and connects the same identifier to all online, print, and electronic versions of the publication. SPIE uses a six-digit CID article numbering system in which:

- The first four digits correspond to the SPIE volume number.

- The last two digits indicate publication order within the volume using a Base 36 numbering

system employing both numerals and letters. These two-number sets start with 00, 01, 02, 03, 04, $05,06,07,08,09,0 A, 0 B \ldots$. OZ, followed by 10-1Z, 20-2Z, etc.

The CID Number appears on each page of the manuscript. The complete citation is used on the first page, and an abbreviated version on subsequent pages. 


\title{
Contents
}

\author{
vii Authors \\ ix Conference Committee
}

\section{SESSION 1 HIGH-ENERGY AND HIGH-POWER DPSSL}

951302 DiPOLE100: A $100 \mathrm{~J}, 10 \mathrm{~Hz}$ DPSSL using cryogenic gas cooled Yb:YAG multi slab amplifier technology (Invited Paper) [9513-1]

951303 High-energy picosecond hybrid fiber/crystal laser for thin films solar cells micromachining [9513-2]

951304 Amplification of picosecond pulses to $100 \mathrm{~W}$ by an Yb:YAG thin-disk with CVBG compressor [9513-3]

951305 High average power picosecond and nanosecond laser operating at 1342nm wavelength [9513-4]

\section{SESSION 2 SHORT-PULSE AND HIGH-PEAK POWER LASERS}

951308 Method of aligning a four-grating compressor for the petawatt-class PEARL-X laser system [9513-7]

9513 OA Multi petawatt laser design for the SHENGUANG II laser facility [9513-9]

9513 OB Timing jitter measurement and stabilization of a mode-locked ytterbium fiber laser [9513-10]

\section{SESSION 3 FRONT ENDS, DEVICES, PUMP SOURCES}

$95130 \mathrm{C}$ Industrial $\mathrm{mJ}$-class all-fiber front end with spatially coherent top-hat beam output used as seeder for high power laser (Invited Paper) [9513-11]

9513 OE Reliable pump sources for high-energy class lasers [9513-13]

9513 OG AIGalnN laser diode bar and array technology for high power and individually addressable applications [9513-15]

$9513 \mathrm{OH} \quad$ AOM optimization with ultra stable high power $\mathrm{CO}_{2}$ lasers for fast laser engraving [9513-16] 
9513 Ol Large aperture adaptive optics for intense lasers (Invited Paper) [9513-17]

9513 0J Alignment system for high-power large aperture laser systems [9513-18]

9513 OK High reflective diffraction grating for ultrafast pulse compression [9513-45]

$9513 \mathrm{OL}$ Thermally induced depolarization in the optical elements of the transition configuration: thick disks and short rods [9513-20]

\section{SESSION 5 NOVEL LASER MATERIALS}

$951300 \quad \mathrm{Yb}: \mathrm{Lu}_{2} \mathrm{SiO}_{5}$ crystal : characterization of the laser emission along the three dielectric axes [9513-22]

9513 OR Graded Yb:YAG ceramic structures: design, fabrication and characterization of the laser performances [9513-25]

9513 OS Investigation of $\mathrm{Yb}^{3+}$-doped alumino-silicate glasses for high energy class diode pumped solid state lasers [9513-26]

\section{SESSION 6 FREQUENCY CONVERSION}

9513 OU Multi-mJ, kHz picosecond deep UV source based on a frequency-quadrupled cryogenic Yb:YAG laser (Invited Paper) [9513-28]

9513 OV Picosesond pulses in deep ultraviolet produced by a $100 \mathrm{kHz}$ solid-state thin disk laser [9513-29]

\section{POSTER SESSION}

$95130 X \quad$ Experimental benchmarking of the code for Yb:YAG multi-slab gas-cooled laser system operating at cryogenic temperatures [9513-19]

9513 OY Thermal distortion real-time detection and correction of a high-power laser beam-splitter mirror based on double Shack-Hartmann wavefront sensors [9513-31]

$95130 Z$ Wavelength-tunable erbium-doped fiber laser using silicon-on-insulator (SOI) based micro-ring with narrow laser linewidth [9513-32]

951310 Active mode control of solid state laser using an intra-cavity beam shaper [9513-33]

951311 Temperature dependent absorption measurement of various transition metal doped laser materials [9513-34] 
951312 Development of a mode-locked fiber laser system for a high finesse enhancement cavity [9513-36]

951313 Demonstration of an optical enhancement cavity with 10 micron wavelength [9513-37]

951314 Multiple pulses and harmonic mode locking from passive mode-locked ytterbium doped fiber in anomalous dispersion region [9513-38]

951316 Sub-picosecond laser induced damage test facility for petawatt reflective optical components characterizations [9513-40]

951317 Generation of 1.6 ns Q-switched pulses based on Yb:YAG/Cr:YAG microchip laser [9513-41]

951318 Research on thermal effects of beam-splitter mirror in high-power laser system [9513-42]

951319 Different mode-locking methods in high energy all-normal dispersion Yb femtosecond allfiber lasers (Best Student Paper Award) [9513-44]

95131 A An intra-cavity device with a discharge-drived CW DF chemical laser [9513-46] 
Proc. of SPIE Vol. $9513951301-6$

Downloaded From: https://www.spiedigitallibrary.org/conference-proceedings-of-spie on 25 Apr 2023 Terms of Use: https://www.spiedigitallibrary.org/terms-of-use 


\section{Authors}

Numbers in the index correspond to the last two digits of the six-digit citation identifier (CID) article numbering system used in Proceedings of SPIE. The first four digits reflect the volume number. Base 36 numbering is employed for the last two digits and indicates the order of articles within the volume. Numbers start with 00, 01, 02, 03, 04, 05, 06, 07, 08, 09, 0A, OB...0Z, followed by 10-1Z, 20-2Z, etc.

Accary, Jean-Baptiste, 0J

Banerjee, Saumyabrata, 02

Beitlerova, Alena, 00

Bertrand, Anthony, 03

Blake, Steve, 02

Boćkowski, Mike, OG

Bohrer, Markus, $\mathrm{OH}$

Boivinet, Simon, 03

Bouwmans, Géraud, OC

Buldt, Joachim, OS

Butcher, Thomas J., 02

Calvet, Pierre, OC

Chang, Chun-Lin, OU

Chekhlov, Oleg, 02

Chow, C. W., $\mathrm{OZ}$

Chyla, Michal, 04

Ciofini, Marco, OR

Collier, John L., 02

Czernecki, Robert, OG

De Vido, Mariastefania, 02

Deneuville, François, 01

Divoky, Martin, 02, 0X

Du, Shaojun, OY, 18

Endo, Akira, 04, OB, OV, 12, 13

Ertel, Klaus, 02

Esposito, Laura, OR

Ferrara, Paolo, OR

Gallais, Laurent, 16

Gao, Qi, OA

Gizzi, Leonida A., OR

Gleyze, Jean-François, OC

Gouriou, Pierre, 0C

Greenhalgh, R. Justin S., 02

Grishin, Mikhail, 05

Guo, Ailin, OA

Hein, Joachim, OS

Hernandez, Yves, 03

Hernandez-Gomez, Cristina, 02

Herrmann, Andreas, OS

Holligan, Paul, 02

Hong, Kyung-Han, OU

Horackova, Lucie, 11

Hostaša, Jan, OR

Hsieh, Wen-Feng, 14

Hugonnot, Emmanuel, OC

Hülsewede, Ralf, OE

Hybler, Jiri, 00

Jambunathan, Venkatesan, 11

Jelínková, Helena, 11, 17
Jiang, Zongfu, 10, 18

Kärtner, Franz X., OU

Kalinchenko, Galina, OK

Kaluza, Malte C., OS

Kang, Jun, OA

Kardaś, Tomasz, 19

Kindsvater, Alex, OE

Kobayashi, T., 12

Körner, Jörg, OS

Kramer, Daniel, OK

Krogen, Peter, OU

Kucharski, Robert, OG

Kuhn, Stefan, OS

Labate, Luca, OR

Lai, Chien-Jen, OU

Lamaignère, Laurent, 16

Lapucci, Antonio, OR

Lavastre, Eric, 16

Lecourt, Jean-Bernard, 03

Lecren, E., OC

Lee, Hung-Yi, 14

LeGarrec, Bruno, 0J

Lekime, Didier, 03

Lerer, Alexander, OK

Leszczyński, Mike, OG

Liang, Houkun, OU

Lin, Ja-Hon, 14

Linnemann, Jens, OB

Liu, Wenguang, 10, 18, 1A

Lu, Qisheng, 1A

Lucianetti, Antonio, OX, 11

Marona, Lucja, OG

Mason, Paul D., 02

Měsíček, Jakub, OB

Meusel, Jens, OE

Michailovas, Andrejus, 05

Michalska, Maria, 19

Miura, Taisuke, 04, OB, OV

Mocek, Tomáš, 04, OB, OV, OX, 11

Moses, Jeffrey, OU

Najda, Stephen P., OG

Néauport, Jérôme, 16

Nejezchleb, Karel, 17

Nikl, Martin, 00

Ning, Yu, OY, 18

Novák, Ondřej, 04, OV

Palashov, O. V., OL

Perlin, Piotr, OG

Perrin, A., OC 
Phillips, P. Jonathan, 02

Piancastelli, Andreana, OR

Pietrzak, Agnieszka, OE

Pilar, Jan, 02

Pirri, Angela, 00, OR

Ploetzeneder, Birgit, 0J

Radzewicz, Czesław, 19

Rodin, Aleksej M., 05

Ropert, Laurent, 01

Roquin, Nadja, 16

Rus, Bedrich, OK

Rüssel, Christian, OS

Sakave, K., 12, 13

Sauvageot, Paul, 01

Sawicka-Chyla, M., OX

Scol, Florent, OC

Sebastian, Jürgen, OE

Seifert, Reinhard, OS

Shaikh, Waseem, 02

Shoji, Yasuhiro, 00

Škoda, Václav, 17

Slezak, O., OX

Smith, Jodie M., 02

Smrž, Martin, 04, OB, OV

Sozet, Martin, 16

Starobor, A. V., OL

Stein, Gregory J., OU

Stepanenko, Yuriy, 19

Šulc, Jan, 11, 17

Sun, Quan, OY, 18

Suski, Tadek, OG

Suzuki, R., 12

Szczepanek, Jan, 19

Targowski, Grzegorz, 0G

Theis, Sébastien, 01

Tiegel, Mirko, OS

Toci, Guido, 0O, OR

Tomlinson, Stephanie, 02

Tsang, H. K., OZ

Turčičová, H., OV

Valentin, Constance, OC

Vannini, Matteo, 0O, OR

Versaci, Roberto, OJ

Vyhlidka, Stepan, OK

Wang, Hongyan, OY

Washio, M., 12, 13

Wisniewski, Przemek, OG

Wolf, Jürgen, OE

Wölz, Martin, OE

Wu, Wuming, OY

$X i e$, Xinglong, $O A$

$X U, X i a o j u n, O Y$

Yakovlev, Ivan, 08

Yan, Baozhu, 10, 1A

Yang, L. G., $0 Z$

Yang, Qingwei, OA

Yeh, C. H., OZ

Yoshikawa, Akira, 00

Yuan, Shengfu, 1A

Yue, Fangxin, OS
Zhou, Qiong, 10, 1A

Zhu, Haidong, OA

Zhu, Jianqiang, OA

Zhu, Ping, OA

Ziano, Roberto, 0J

viii

Proc. of SPIE Vol. $9513951301-8$ 


\title{
Conference Committee
}

\author{
Symposium Chairs
}

Jiri Homola, Institute of Photonics and Electronics of the ASCR, v.v.i.

(Czech Republic)

Chris Edwards, Central Laser Facility, Science and Technology Facilities Council (United Kingdom)

Mike Dunne, SLAC National Accelerator Laboratory (United States) and Linac Coherent Light Source (United States)

Ivo Rendina, Istituto per la Microelettronica e Microsistemi, CNR (Italy)

Honorary Symposium Chair

Miroslav Miller, Institute of Photonics and Electronics of the ASCR, v.v.i. (Czech Republic)

\section{Conference Chair}

Joachim Hein, Friedrich-Schiller-Universität Jena (Germany)

Conference Program Committee

Jean-Christophe Francis Chanteloup, École Polytechnique (France)

Leonida A. Gizzi, Consiglio Nazionale delle Ricerche (Italy)

Marc Hanna, Laboratoire Charles Fabry (France)

Bruno J. Le Garrec, Institute of Physics of the ASCR, v.v.i. (Czech Republic)

Jens Limpert, Friedrich-Schiller-Universität Jena (Germany)

Antonio Lucianetti, Institute of Physics of the ASCR, v.v.i. (Czech Republic)

Paul D. Mason, Rutherford Appleton Laboratory (United Kingdom)

Mathias Siebold, Helmholtz-Zentrum Dresden-Rossendorf e. V.

(Germany)

Session Chairs

$1 \quad$ High Energy and High Power DPSSL

Joachim Hein, Friedrich-Schiller-Universität Jena (Germany)

2 Short Pulse and High Peak Power Lasers

Paul D. Mason, Rutherford Appleton Laboratory (United Kingdom) 
3 Front Ends, Devices, Pump Sources

Sebastian Keppler, Friedrich-Schiller-Universität Jena (Germany)

4 Large Aperture Optics and Thermal Problems

Jean-François Gleyze, Commissariat à l'Énergie Atomique (France)

5 Novel Laser Materials

Laurent Ropert, ISP System (France)

6 Frequency Conversion

Guido Toci, Consiglio Nazionale delle Ricerche (Italy) 